\title{
Metabolic alterations in the rat cerebellum following acute middle cerebral artery occlusion, as determined by ${ }^{1} \mathrm{H}$ NMR spectroscopy
}

\author{
ZI-LONG HU ${ }^{1 *}$, HUAN-HUAN XIA $^{2,3^{*}}$, YUN-JUN YANG ${ }^{1 *}$, HONG ZHENG $^{2}$, LIANG-CAI ZHAO ${ }^{2}$, \\ YONG-CHUN CHEN ${ }^{1}$, QI-CHUAN ZHUGE ${ }^{1}$, NENG-ZHI XIA ${ }^{1}$, HONG-CHANG GAO ${ }^{2}$ and WEI-JIAN CHEN ${ }^{1}$ \\ ${ }^{1}$ Department of Radiology, The First Affiliated Hospital of Wenzhou Medical University/Zhejiang Province \\ Key Laboratory of Aging and Neurological Disorder Research; ${ }^{2}$ Institute of Metabonomics and Medical NMR, \\ School of Pharmaceutical Sciences, Wenzhou Medical University, Wenzhou, Zhejiang 325000; ${ }^{3}$ Department of \\ Pharmacy, The Third Affiliated Hospital of Wenzhou Medical University, Rui'an, Zhejiang 325200, P.R. China
}

Received June 24, 2016; Accepted September 11, 2017

DOI: $10.3892 / \mathrm{mmr} .2017 .7918$

\begin{abstract}
Supratentorial focal ischemia may reduce cerebral blood volume and cerebellar glucose metabolic rate contralateral to the region of ischemia. The present study investigated the effects of middle cerebral artery occlusion (MCAO) on cerebral metabolism in the ischemic cerebral hemisphere and the non-ischemic cerebellum in rats 1,3,9 and $24 \mathrm{~h}$ following ischemia using ex vivo proton nuclear magnetic resonance ( ${ }^{1} \mathrm{H}$ NMR) spectroscopy. The results demonstrated that focal ischemia induced increases in the levels of lactate and alanine, and a decrease in succinate, as early as $1 \mathrm{~h}$ following ischemia in the left cerebral hemisphere and the right cerebellum. A continuous increase in lactate levels and
\end{abstract}

Correspondence to: Dr Wei-Jian Chen, Department of Radiology, The First Affiliated Hospital of Wenzhou Medical University/Zhejiang Province Key Laboratory of Aging and Neurological Disorder Research, 2 Fuxue Lane, Wenzhou, Zhejiang 325000, P.R. China

E-mail:wzfskyyj2011@163.com

Dr Hong-Chang Gao, Institute of Metabonomics and Medical NMR, School of Pharmaceutical Sciences, Wenzhou Medical University, University Town, 106 Nanliu Road, Wenzhou, Zhejiang 325000, P.R. China

E-mail: gaohc27@wmu.edu.cn

*Contributed equally

Abbreviations: MCAO, middle cerebral artery occlusion; ${ }^{1} \mathrm{H}$ NMR, proton nuclear magnetic resonance; $\mathrm{CCD}$, crossed cerebellar diaschisis; TCA, tricarboxylic acid; CCA, common carotid artery; ECA, external carotid artery; ICA, internal carotid artery; PLS-DA, partial least squares discriminate analysis; NAA, N-acetyl aspartate; Lac, lactate; Ala, alanine; GABA, $\gamma$-aminobutyric acid; Glu, glutamate; Gln, glutamine; Suc, succinate; Asp, aspartate; Cre, creatine; m-Ins, myo-inositol; Cho, choline; Gly, glycine; ${ }^{1} \mathrm{H}$ MRS, ${ }^{1} \mathrm{H}$ magnetic resonance spectroscopy

Key words: middle cerebral artery occlusion, ischemia, rat, cerebellum, metabolites, ${ }^{1} \mathrm{H}$ NMR decrease in creatine levels were detected in both cerebral areas 3 and 24 h post-MCAO. The most obvious difference between the two cerebral areas was that there was no statistically significant difference in $\mathrm{N}$-acetyl aspartate (NAA) levels in the right cerebellum at all time points; however, the amino acid levels of NAA in the left cerebral hemisphere were markedly decreased 3, 9 and 24 h post-MCAO. In addition, an obvious increase in glutamine was observed in the right and left cerebellum at 3,9 and 24 h post-MCAO. Furthermore, the present study demonstrated that $\gamma$-aminobutyric acid levels were decreased at $1 \mathrm{~h}$ in the left and right cerebellum and were evidently increased at $24 \mathrm{~h}$ in the right cerebellum post-MCAO. In conclusion, supratentorial ischemia has been indicated to affect the activities of the non-ischemic contralateral cerebellum. Therefore, these results suggested that an NMR-based metabonomic approach may be used as a potential means to elucidate cerebral and cerebellar metabolism following MCAO, which may help improve understanding regarding cerebral infarction at a molecular level. Ex vivo ${ }^{1} \mathrm{H}$ NMR analysis may be useful for the assessment of clinical biopsies.

\section{Introduction}

Acute ischemic stroke is a type of focal brain injury, which causes functional depression due to a disruption in normal signal propagation between the ischemic area and regions that are connected to it by nerve fiber bundles (1). Crossed cerebellar diaschisis (CCD), which was first mentioned by Baron et al (2), is a condition in which blood flow and metabolism on the side contralateral to a damaged cerebral area are decreased (3). It has previously been reported that an interruption in corticopontocerebellar pathways is the most likely mechanism underlying CCD (3-5). Following a supratentorial stroke, cortical excitability cannot be transmitted to the contralateral cerebellum due to pathway disruption, which leads to functional inhibition and a decrease in metabolism in the contralateral cerebellar hemisphere (6). To date, numerous techniques, including single photon emission computed tomography, positron emission tomography, dynamic susceptibility contrast magnetic resonance (MR) perfusion imaging, 
arterial spin-labeling MR imaging and computed tomography perfusion, have been used to estimate CCD within stroke patients (7-14). These techniques attempt to diagnose CCD based on the rate of regional cerebral blood flow and oxygen metabolism in the brain; however, the mechanisms underlying CCD remain unclear.

Regional metabolic differences in the mammalian brain, including glucose and glycogen stores, have been detected in ex vivo analyses (15), and have also been determined from non-invasive measurements in humans and mice (16-18). Håberg et al (1) reported that glucose metabolism and the metabolic activity of intermediates from the astrocytic tricarboxylic acid (TCA) cycle were markedly decreased in the whole rat cerebellum in the superacute stage of middle cerebral artery occlusion (MCAO), as determined using ${ }^{13} \mathrm{C} \mathrm{MR}$ spectra. In addition, it was demonstrated that the cerebellum could control hemispheric activity. Therefore, it may be suggested that, to enhance the recovery of cerebral hemispheric function, it would be beneficial to maintain the cerebellum in a low-activity state. However, this previous study did not explore metabolism in the bilateral cerebellum or observe variations between left and right sides. Previous studies have reported that CCD may not be just a concomitant phenomenon of stroke, but may be regarded as a crucial prognostic indicator, which may benefit the treatment and rehabilitation of brain ischemia $(10,19)$.

The present study aimed to identify the effects of MCAO on alterations in cerebral metabolism in the ischemic brain regions and in the contralateral cerebellum in rats $1,3,9$ and $24 \mathrm{~h}$ following ischemia using proton nuclear MR ( ${ }^{1} \mathrm{H}$ NMR) spectroscopy. In addition, the study aimed to: i) Evaluate the regional metabolic differences induced by CCD between the ischemic cerebral hemisphere and the contralateral cerebellum; and ii) to identify the mechanisms underlying CCD.

\section{Materials and methods}

Animal preparation and treatment. A total of 38 male Sprague-Dawley rats (8-10 weeks old; 250-320 g; Shanghai SLAC Laboratory Animal Co., Ltd., Shanghai, China) were maintained in the Specific-Pathogen-Free Animal Experimental Center of Wenzhou Medical University (Wenzhou, China). All rats were kept under a temperature of $23 \pm 20^{\circ} \mathrm{C}$ and a relative humidity of $55 \pm 10 \%$, and were maintained under a 12-h light/dark cycle with free access to food and drink. The present study was approved by the Animal Ethics Committee of Wenzhou Medical University and was strictly conducted according to the National Institutes of Health Guide for the Care and Use of Laboratory Animals (20).

Development of the MCAO model. An MCAO model was developed using the intraluminal filament technique, as previously described (21). After $12 \mathrm{~h}$ of fasting, the rats were anesthetized with $10 \%$ chloral hydrate $(300 \mathrm{mg} / \mathrm{kg}$; intraperitoneal). Initially, an incision was made into the middle cervical fascia and the left common carotid artery (CCA) was exposed. The external carotid artery (ECA) and internal carotid artery (ICA) were then separated. Subsequently, the bifurcation close to the ECA was ligated with the filament. A ready-made suture (Beijing Sunbio Biotech Co., Ltd., Beijing, China) was inserted via the left CCA into the ICA, in order to occlude the MCA. The depth of the suture within the vessel was 16-18 $\mathrm{mm}$ and the redundant part was cut off with a ligature. In this procedure, the suture was maintained around the vessel. Finally, the incision was stitched and the rats were fed separately to improve survival rate. The temperature was maintained at $25-26^{\circ} \mathrm{C}$ during the surgery. In the sham operation group $(n=10)$, the neck was incised to expose the left CCA; however, the MCA was not occluded. MCAO rats $(n=28)$ were randomly sacrificed by prompt decapitation at the following time points: $1,3,9$ and $24 \mathrm{~h}$ after MCAO ( $\mathrm{n}=7 /$ group). The rats in the sham operation group were decapitated $24 \mathrm{~h}$ after surgery and were compared with the MCAO rats at all other time points. Tissue specimens were obtained from the left cerebral hemisphere, and the left and right cerebellum, within $15 \mathrm{sec}$; tissue specimens were frozen at $-80^{\circ} \mathrm{C}$.

Preparation of cerebral samples. The frozen brain tissues were weighed and homogenized in centrifuge tubes using an electric homogenizer. The samples were then vortexed with $4 \mathrm{ml} / \mathrm{g}$ ice-cold methanol and $0.85 \mathrm{ml} / \mathrm{g}$ distilled water. Subsequently, $2 \mathrm{ml} / \mathrm{g}$ chloroform and $2 \mathrm{ml} / \mathrm{g}$ distilled water were added to the tubes and mixed again. The specimen tubes were placed on ice for $15 \mathrm{~min}$ and were then centrifuged at $12,000 \mathrm{x} \mathrm{g}$ for $15 \mathrm{~min}$ at $4^{\circ} \mathrm{C}$. The supernatant was separated from the tubes and placed into a freeze-dryer to lyophilize for $24 \mathrm{~h}$. Finally, the obtained extracts were dissolved in $500 \mu 199.5 \% \mathrm{D}_{2} \mathrm{O}$ for NMR spectroscopy.

Acquisition of ${ }^{1} \mathrm{H}$ NMR spectra. All ${ }^{1} \mathrm{H}$ NMR spectra of the extracts were obtained at $25^{\circ} \mathrm{C}$ with a $90^{\circ}$ flip angle on a spectrometer (Bruker Avance III 600-MHz; Bruker Corporation, Billerica, MA, USA). The spectral width was set at $12,000 \mathrm{~Hz}$ and $32 \mathrm{~K}$ data points. The collection time was $2.66 \mathrm{sec}$ per scan and the number of scans was 128 . In order to assure full relaxation, an extra $8 \mathrm{sec}$ relaxation delay was set. Exponential line-broadening of $0.3 \mathrm{~Hz}$ was used in the free induction decay ahead of Fourier transformation and the spectra were zero-filled to $64 \mathrm{~K}$. All spectra were carefully corrected by hand for phase as well as baseline. In addition, the methyl peak of lactate (Lac) $\left(\mathrm{CH}_{3} ; 1.33 \mathrm{ppm}\right)$ was used as a reference point for the spectra. Peak area integration was conducted using the Bruker Topspin software package (version 2.1; Bruker Corporation) with standard routines.

Data and statistical analysis. NMR spectra (\$0.5-10.0) were segmented into integral intervals with each width of $0.01 \mathrm{ppm}$ (2.4 Hz) through AMIX package (Bruker Topspin 2.1; Bruker Corporation), so that all metabolic information embedded in the spectra could be exploited; the sum of each spectrum was then standardized. The normalized integral values were mean-centered for multivariate data analysis using software (Umetrics SIMCA-P+12.0; Sartorius Stedim Data Analytics AB, Umeå, Sweden). Partial least squares discriminate analysis (PLS-DA) was conducted to identify metabolites according to the separation of different groups (22). Data were visualized using a principal component scores plot of the first two principal components to provide the most efficient 2D representation of the information (20). Data were confirmed in $2 \mathrm{D}{ }^{1} \mathrm{H}-{ }^{1} \mathrm{H}$ correlation spectroscopy and total correlation 
spectroscopy spectra. The position of each point represents an individual spectrum of a sample. Differences in the sample compositions between the different groups were determined by PLS-DA, and differences in the metabolites between the groups were revealed as coefficient of variation plots (23). Model quality was assessed with the fitness of model $\left(\mathrm{R}^{2}\right)$ and the predictive ability of model $\left(\mathrm{Q}^{2}\right)$.

All values are presented as the mean \pm standard deviation. SPSS software (version 13.0; SPSS, Inc., Chicago, IL, USA) was used to determine the statistical differences between groups using one-way analysis of variance followed by a least significant difference post hoc test for multiple comparisons. $\mathrm{P}<0.05$ was considered to indicate a statistically significant difference.

\section{Results}

${ }^{1} \mathrm{H}$ NMR spectral analysis of samples. ${ }^{1} \mathrm{H}$ NMR was used to investigate metabolism within the contralateral side to the damaged area; the typical ${ }^{1} \mathrm{H}$ NMR spectra of the right rat cerebellum samples in the sham operation, and 1,3,9 and $24 \mathrm{~h}$ MCAO model groups are presented in Fig. 1. The allocation of metabolites on the spectrograms was based on our previous work (24). 2D ${ }^{1} \mathrm{H}-{ }^{1} \mathrm{H}$ correlation spectroscopy and total correlation spectroscopy of the representative samples were performed to confirm the allocations on the ${ }^{1} \mathrm{H}$ NMR spectra. Numerous endogenous metabolites were simultaneously observed on the ${ }^{1} \mathrm{H}$ NMR spectra of cerebral samples.

Pattern recognition of cerebral extracts. The ${ }^{1} \mathrm{H}$ NMR data of the left cerebral hemisphere and the right cerebellum were used to determine differences between metabolic profiling of rats in the MCAO and sham groups after 1, 3, 9 and 24 h by multivariate data analysis. PLS-DA score plots displayed a prominent separation between the sham and MCAO groups along the direction of $t(1)$ in the left hemisphere (Fig. 2) and the right cerebellum (Fig. 3A), revealing a significant metabolic disturbance. However, with the increase in ischemic duration, the alteration in metabolic patterns moved gradually away from the $t(1)$ direction in the left cerebral hemisphere (Fig. 2), which was not detected in the right cerebellum at $9 \mathrm{~h}$ (Fig. 3B). Such alterations indicated that the two brain regions have differences in metabolic pattern.

The comparisons between rats in the sham and $1 \mathrm{~h}$ model groups, and the sham and $24 \mathrm{~h}$ model groups are presented in Figs. 4 and 5, respectively. The PLS-DA score plots of the sham operation group and the $1 \mathrm{~h}$ model group, and the sham operation group and the $24 \mathrm{~h}$ model group are presented in Figs. 4A and 5A, respectively. The model groups may be separated from the sham operation group along the first principal components horizontal direction. The results demonstrated that there was an obvious difference between the two groups with regards to the spectral features in the right cerebellum. In addition, the validation graph of permutation tests indicated that the PLS-DA models were robust and credible (Figs. 4B and $5 \mathrm{~B})$.

Figs. 4C and 5C illustrate the corresponding loading plots of metabolites between the MCAO and sham groups using color-coded correlation coefficients at 1 and $24 \mathrm{~h}$ in the right cerebellum. The findings indicated that the separation of the

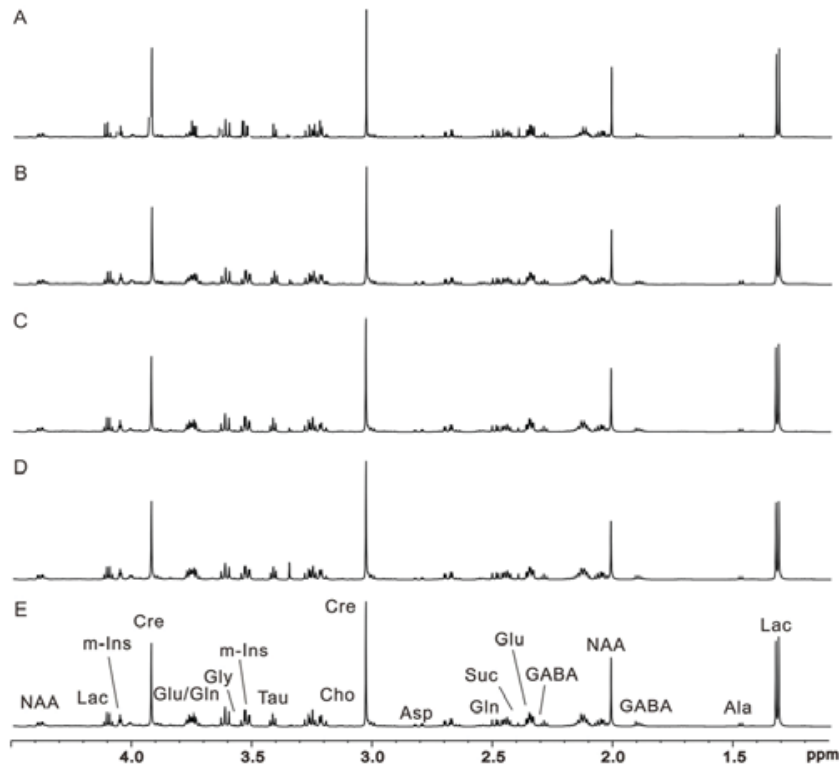

Figure 1. Representative $600-\mathrm{MHz}$ proton nuclear magnetic resonance spectra of right cerebellum extracts obtained from rats in (A-D) the model groups (1, 3, 9 and $24 \mathrm{~h}$ after middle cerebral artery occlusion, respectively) and (E) the sham operation group. Ala, alanine; Asp, aspartate; Cre, creatine; Cho, choline; GABA, $\gamma$-aminobutyric acid; Gln, glutamine; Glu, glutamate; Lac, lactate; m-Ins, myo-inositol; NAA, N-acetyl aspartate; Suc, succinate.

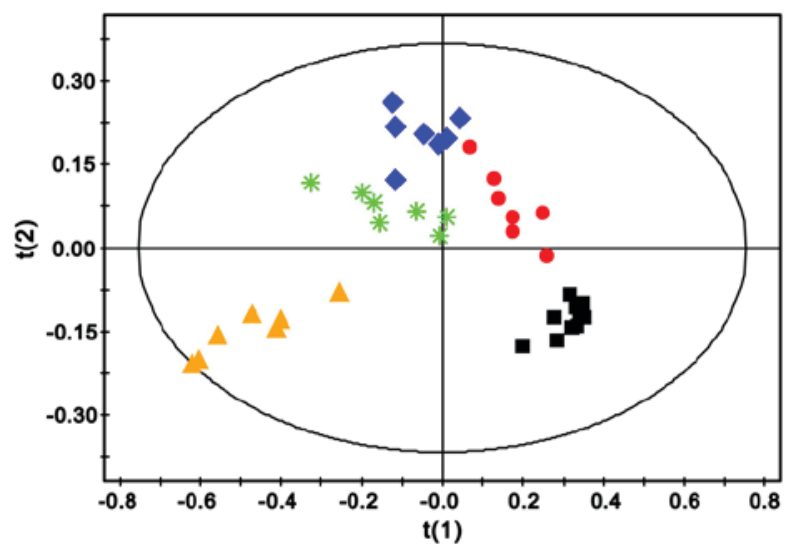

Figure 2. Partial least squares discriminate analysis score plots of the first and second principal components based on the proton nuclear magnetic resonance spectra of the left cerebral hemisphere samples obtained from rats in the sham operation (*), MCAO $1 \mathrm{~h}(\bullet)$, MCAO $3 \mathrm{~h}(\bullet)$, MCAO $9 \mathrm{~h} \mathrm{(}\left(^{*}\right)$ and MCAO 24 h $(\boldsymbol{\Delta})$ groups. MCAO, middle cerebral artery occlusion.

different groups may be due to variation in metabolite levels. The square of the correlation coefficient was used as the weight of a variable, and color-coding was used to indicate low and high values (low, blue; high, red). An increase in the corresponding metabolites in MCAO rats was displayed in the negative area, whereas a decrease in the corresponding metabolites was displayed in the positive area. The results demonstrated that MCAO rats had lower levbels of N-acetyl aspartate (NAA), creatine (Cre), glutamate (Glu) and succinate (Suc), and higher levels of lactate (Lac), $\gamma$-aminobutyric acid (GABA) and glutamine (Gln) compared with the control groups. The results at the other time points are presented in Figs. 6 and 7, and were in accordance with those presented in Figs. 4 and 5. 
A

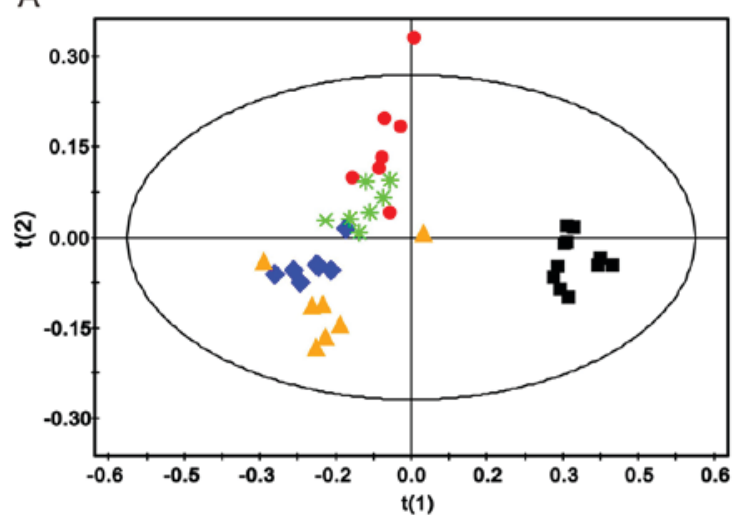

B

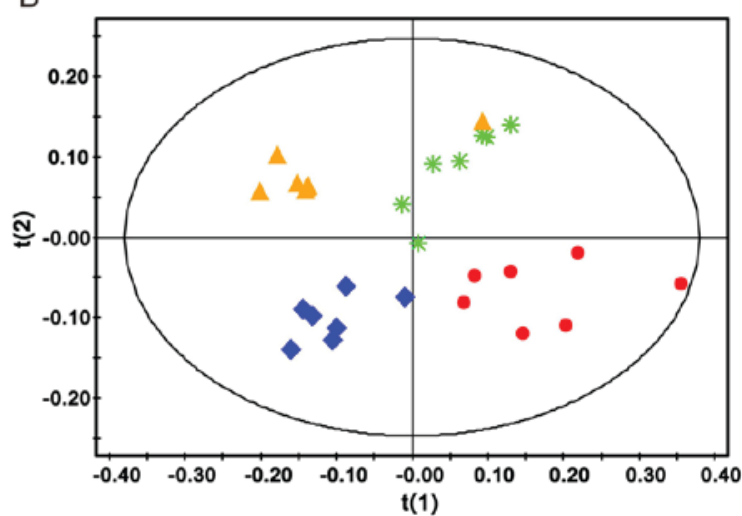

Figure 3. (A) Partial least squares discriminate analysis score plots of the first and second principal components based on the proton nuclear magnetic reso-

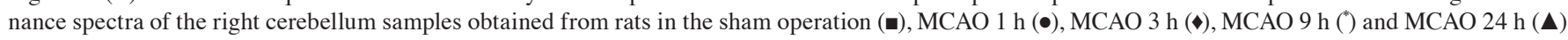
groups. (B) Partial least squares discriminate analysis score plots of the first and second principal components based on the proton nuclear magnetic resonance spectra of the right cerebellum samples obtained from rats in the MCAO $1 \mathrm{~h}(\bullet), \mathrm{MCAO} 3 \mathrm{~h}(\bullet)$, MCAO $9 \mathrm{~h}\left({ }^{*}\right)$ and MCAO $24 \mathrm{~h}(\mathbf{\Delta})$ groups. MCAO, middle cerebral artery occlusion.
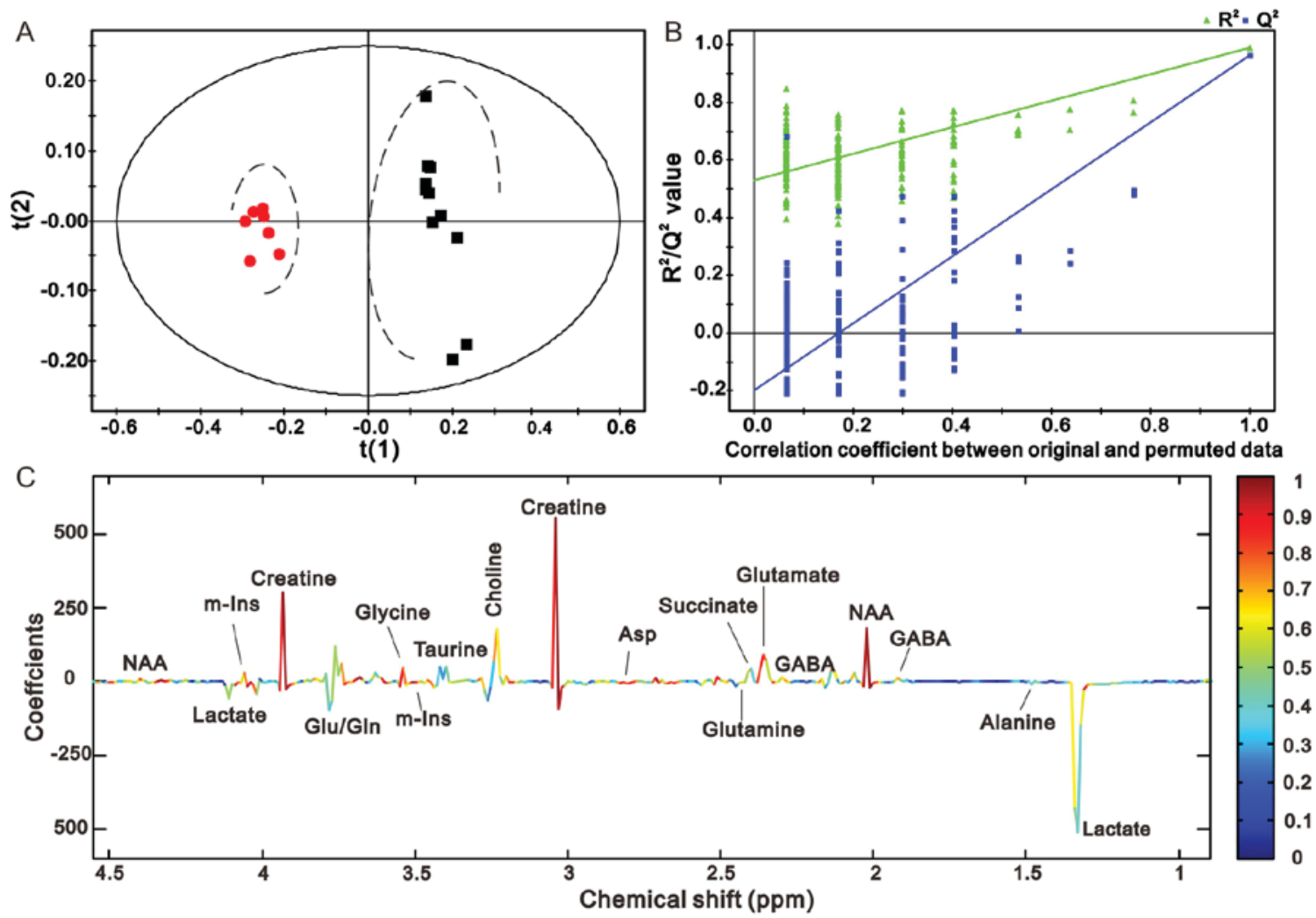

Figure 4. (A) PLS-DA score plot $\left(\mathrm{R}^{2}=0.099, \mathrm{Q}^{2}=0.97\right)$ and (B) validation plot based on the proton nuclear magnetic resonance spectra of right cerebellum samples obtained from rats in the middle cerebral artery occlusion $1 \mathrm{~h}(\bullet)$ and sham operation ( $\bullet$ ) groups. (C) Coefficient-coded loading plot corresponding to the PLS-DA plot revealed that metabolites with varying intensities between the groups were responsible for the separation between the groups in the corresponding score plot. Asp, aspartate; GABA, $\gamma$-aminobutyric acid; m-Ins, myo-inositol; Gln, glutamine; Glu, glutamate; NAA, N-acetyl aspartate; PLS-DA, partial least squares discriminate analysis.

Alterations in the levels of cerebral metabolites. Metabolite levels were quantified, in order to investigate the metabolic alterations in left cerebral tissue (Fig. 8). The results demonstrated that the levels of GABA, glycine (Gly), choline (Cho), Lac and alanine (Ala) were markedly increased in the ischemic cerebral hemisphere of rats compared with in the control group. The levels of Gln were not markedly increased until $9 \mathrm{~h}$ post-MCAO. The levels of Glu, aspartate (Asp),
NAA and Cre were markedly decreased in the left cerebral hemisphere 3,9 and $24 \mathrm{~h}$ post-MCAO. In addition, the levels of Suc were decreased $24 \mathrm{~h}$ post-MCAO, and an obvious decrease in myo-inositol (m-Ins) levels were also detected $24 \mathrm{~h}$ after ischemic insult in the left cerebral hemisphere.

Conversely, ischemia induced marked increases in the levels of Gln, Asp and Lac, and concomitant decreases in the levels of Suc and Cre in the right cerebellum at all studied 
A

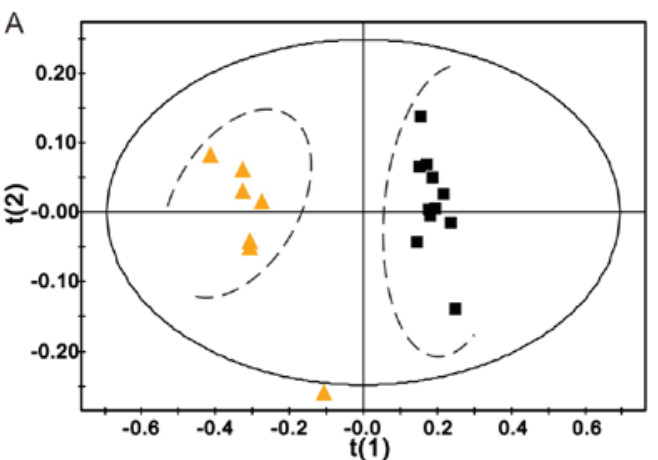

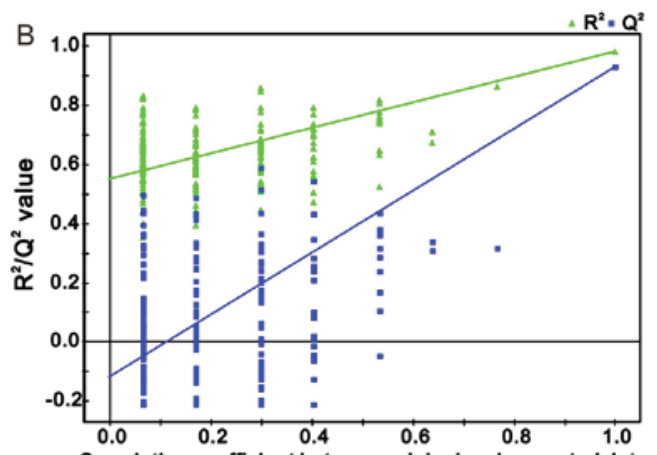

Correlation coefficient between original and permuted data

C

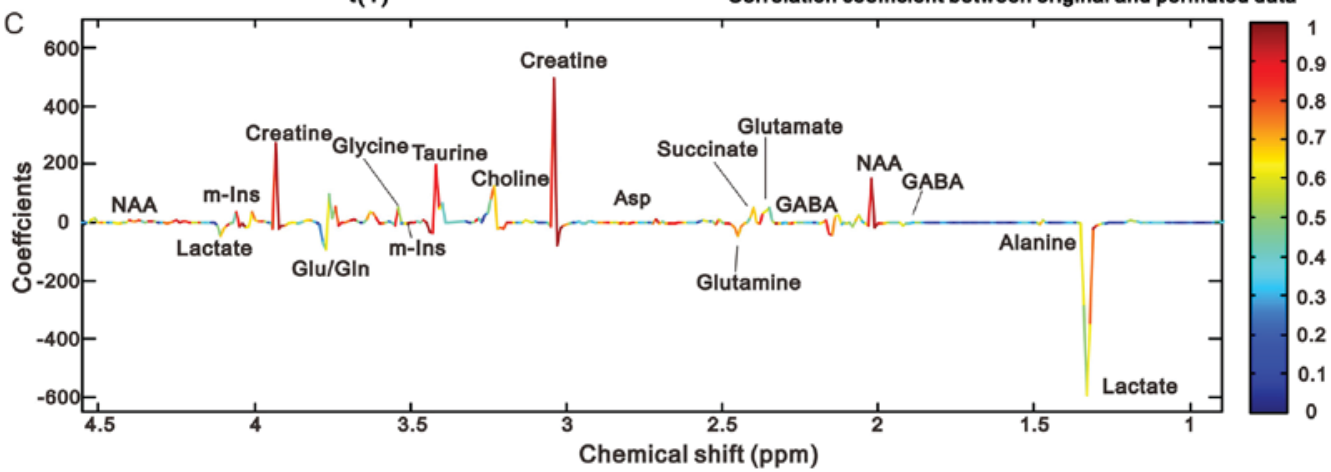

Figure 5. (A) PLS-DA score plot $\left(\mathrm{R}^{2}=0.98, \mathrm{Q}^{2}=0.93\right)$ and (B) validation plot based on the proton nuclear magnetic resonance spectra of right cerebellum samples obtained from rats in the middle cerebral artery occlusion $24 \mathrm{~h}(\boldsymbol{\Delta})$ and sham operation ( $\mathbf{\square})$ groups. (C) Coefficient-coded loading plot corresponding to the PLS-DA plot revealed that metabolites with varying intensities between the groups were responsible for the separation between the groups in the corresponding score plot. Asp, aspartate; GABA, $\gamma$-aminobutyric acid; m-Ins, myo-inositol; NAA, N-acetyl aspartate; PLS-DA, partial least squares discriminate analysis.
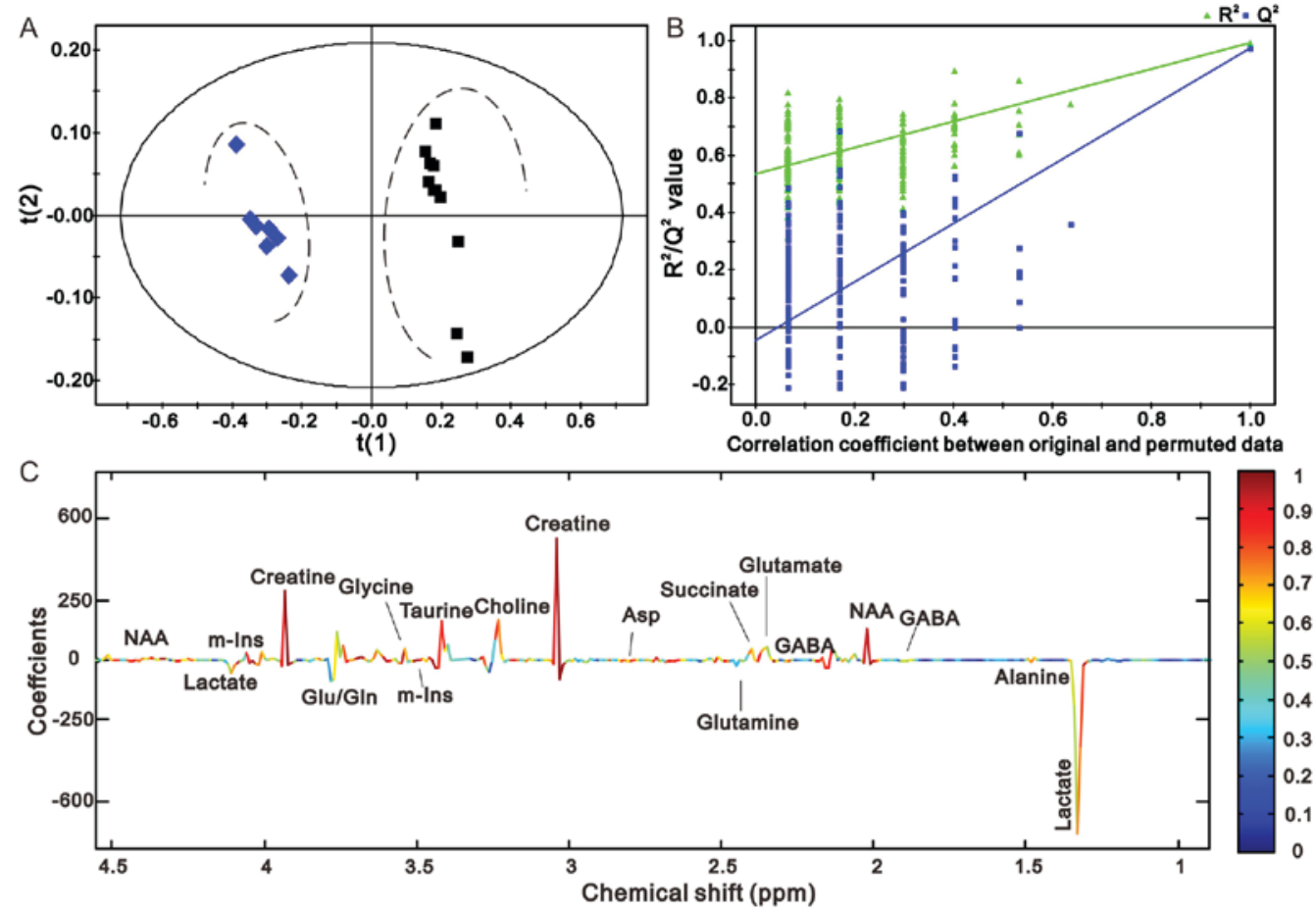

Figure 6. (A) PLS-DA score plot $\left(\mathrm{R}^{2}=0.99, \mathrm{Q}^{2}=0.97\right)$ and (B) validation plot based on the proton nuclear magnetic resonance spectra of right cerebellum samples obtained from rats in the middle cerebral artery occlusion $3 \mathrm{~h}(\bullet)$ and sham operation ( $\bullet$ ) groups. (C) Coefficient-coded loading plot corresponding to the PLS-DA plot revealed that metabolites with varying intensities between the groups were responsible for the separation between the groups in the corresponding score plot. Asp, aspartate; GABA, $\gamma$-aminobutyric acid; m-Ins, myo-inositol; NAA, N-acetyl aspartate; PLS-DA, partial least squares discriminate analysis.

time points post-MCAO (Fig. 9); however, there were no significant differences in NAA levels between the groups (data not shown). Cho levels were not markedly increased until $24 \mathrm{~h}$ post-MCAO. In addition, Ala levels were increased 

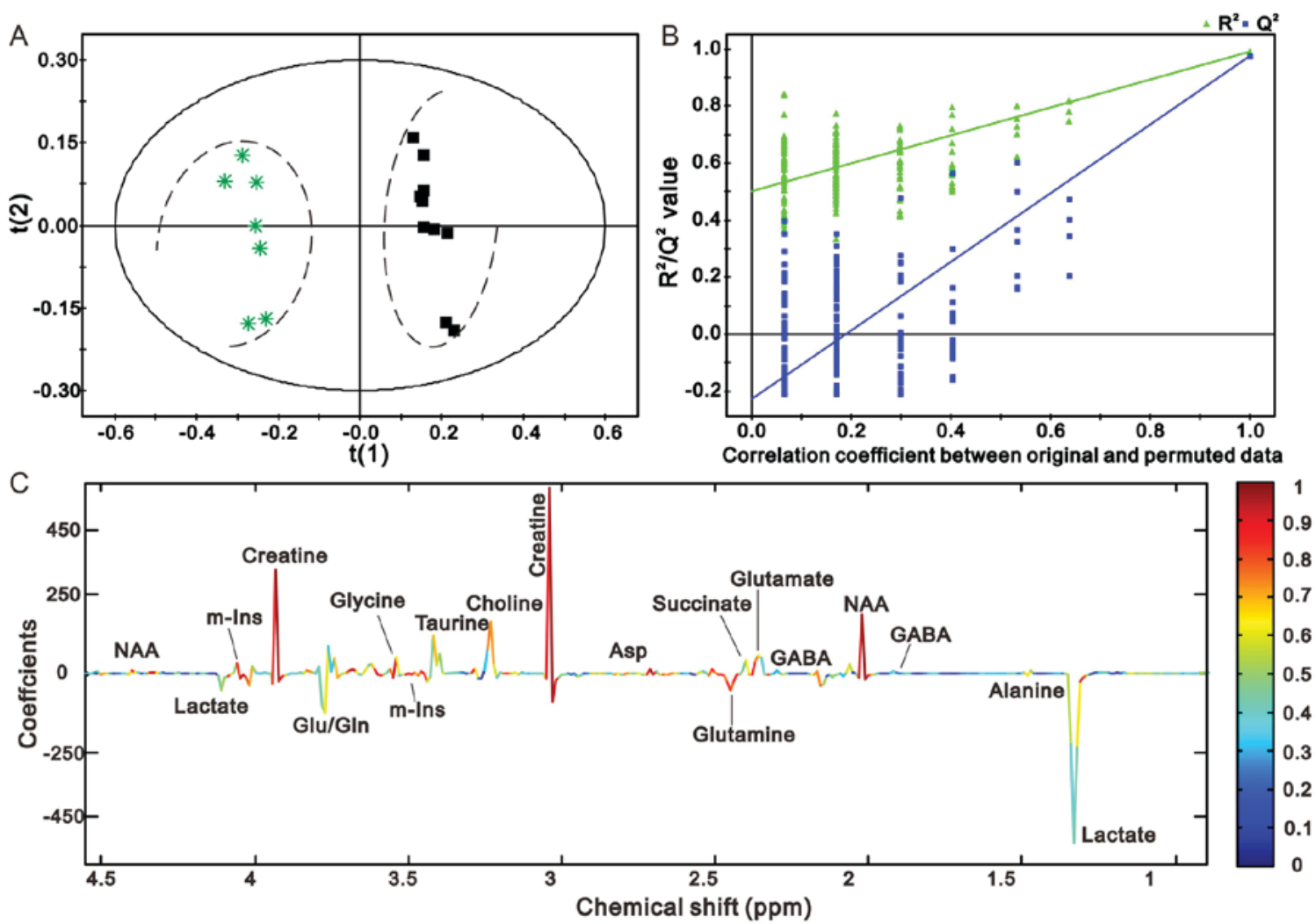

Figure 7. (A) PLS-DA score plot $\left(\mathrm{R}^{2}=0.99, \mathrm{Q}^{2}=0.97\right)$ and (B) validation plot based on the proton nuclear magnetic resonance spectra of right cerebellum samples obtained from rats in the middle cerebral artery occlusion $9 \mathrm{~h} \mathrm{(")} \mathrm{and} \mathrm{sham} \mathrm{operation} \mathrm{(-)} \mathrm{groups.} \mathrm{(C)} \mathrm{Coefficient-coded} \mathrm{loading} \mathrm{plot} \mathrm{corresponding} \mathrm{to} \mathrm{the} \mathrm{PLS-DA}$ plot revealed that metabolites with varying intensities between the groups were responsible for the separation between the groups in the corresponding score plot. Asp, aspartate; GABA, $\gamma$-aminobutyric acid; m-Ins, myo-inositol; NAA, N-acetyl aspartate; PLS-DA, partial least squares discriminate analysis.

at $1 \mathrm{~h}$ post-MCAO, and GABA levels were elevated at 3 and $24 \mathrm{~h}$ following ischemic insult. Conversely, Glu levels were significantly decreased at 1 and $3 \mathrm{~h}$ post-MCAO.

As presented in Fig. 10, there were variations in Glu, Gln, GABA and total levels (Gln + Glu + GABA), and the Glu/GABA and $\mathrm{Glu} / \mathrm{Gln}$ ratios in the non-ischemic left cerebellum and right cerebellum $1,3,9$, and $24 \mathrm{~h}$ post-MCAO and sham operation. The alterations in Glu/Gln ratio and Gln + Glu + GABA had just the same trend between the right and left cerebellum; however, for Glu and Glu/GABA ratio, a similar trend was observed between the right and left cerebellums. A marked increase in Gln levels and a concomitant decrease in the Glu/Gln ratio was detected in both regions at all studied time points post-MCAO. In addition, an obvious increase in Gln was observed in the right and left cerebellum at 3,9 and $24 \mathrm{~h}$ post-MCAO. Furthermore, the present study demonstrated that GABA levels were decreased at $1 \mathrm{~h}$ in the left and right cerebellum and were evidently increased at $24 \mathrm{~h}$ in the right cerebellum post-MCAO.

\section{Discussion}

Since the brain has high sensitivity to ischemic hypoxia, focal ischemia can result in a reduced supply of glucose and oxygen to the corresponding brain areas, resulting in disruption to the TCA cycle (25). Consequently, the levels of Lac, as the main product of anaerobic glycolysis, were markedly increased in the present study in response to ischemia, which is in agreement with the results of previous studies (26-29).
${ }^{1} \mathrm{H}$ MR spectroscopy (MRS) has been widely used to research the pathological mechanism underlying neuronal and cerebral metabolic alterations in response to cerebral ischemia in humans and animals $(26,30,31)$. Alterations in the spectral peaks of patients with acute ischemic stoke may have prognostic value in clinical practice $(32,33)$.

The present study aimed to use ${ }^{1} \mathrm{H}$ NMR spectroscopy to analyze the metabolic alterations of $\mathrm{CCD}$ between the left cerebral hemisphere and the contralateral cerebellum in rats after permanent MCAO. The main finding of the present study was that metabolic alterations were detected in the contralateral cerebellum, which is a region involved in CCD, as determined using PLS-DA. The results indicated that: i) Focal ischemia induced marked increases in the levels of Lac, Ala, Gln and GABA, and decreases in the levels of Cre, Suc and Glu in the left cerebral hemisphere and the right cerebellum; ii) supratentorial ischemia induced metabolic alterations between left and right cerebellum, particularly in the contralateral cerebellum, iii) alterations in Glu metabolism may be associated with CCD; however, further studies are required.

Glu and Asp are the major excitatory amino acids, which have significant roles in the central nervous system. In previous studies, dynamic equilibrium of excitatory and inhibitory amino acids (Glu and GABA) was elevated to the highest level 1-2 $\mathrm{h}$ after ischemia; however, it was decreased $3 \mathrm{~h}$ after ischemic injury in rats $(34,35)$. The present study detected alterations in the levels of Glu, Asp, NAA and Cre at 3,9 and $24 \mathrm{~h}$ in the brain of ischemic rats. In addition, GABA and Gly levels were 

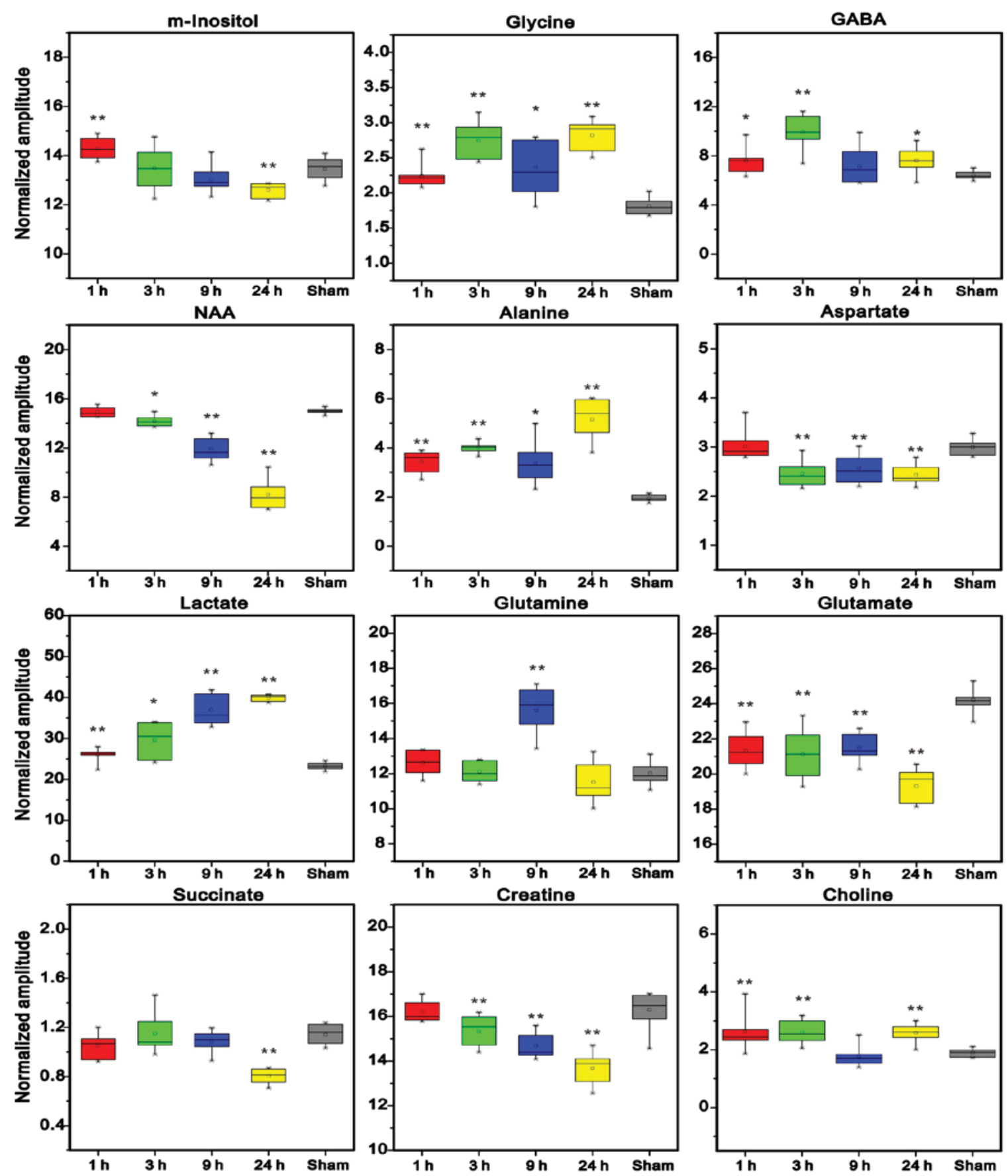

Figure 8. Relative normalized concentrations of the significantly altered metabolites in the left ischemic cerebral hemisphere. Red, green, blue, yellow and grey represent relative normalized concentrations in the middle cerebral artery occlusion 1,3,9 and $24 \mathrm{~h}$, compared with sham operation groups, respectively. ${ }^{*} \mathrm{P}<0.05 ;{ }^{* *} \mathrm{P}<0.01$ vs. the sham group. GABA, $\gamma$-aminobutyric acid.

significantly increased at 1,3 and $24 \mathrm{~h}$ post-MCAO, which indicated that GABA and Gly had protective effects on ischemic insult in the rat brain against excitatory amino acid toxicity.

Glu is a neurotransmitter that is released by neurons and can be absorbed by astrocytes, where it is converted into Gln. Subsequently, Gln can be transferred to neurons and once again converted to Glu. Furthermore, Gln is a major precursor of neuronal Glu and GABA. This important circulatory pathway between astrocytes and neurons is known as the Gln-Glu-GABA cycle (36). Elevated levels of GABA and Gln, and decreased levels of Glu and Asp, were observed in the ischemic cerebral hemisphere of MCAO rats in the present study, which was consistent with the results of a previous study (37). A recent study indicated that the potential mechanisms underlying a decrease in Glu levels may be associated with increased utilization and decreased synthesis (1). Furthermore, Glu may undergo retrograde transport in axons (38). Therefore, it may be hypothesized that excitotoxic action in the ischemic region is increased if Glu is transported from the non-ischemic cerebellum to the ischemic cerebral hemisphere. In the present study, Glu concentrations were significantly decreased in the left cerebral hemisphere 1,3,9 and $24 \mathrm{~h}$ post-MCAO; however, in the right cerebellum Glu was markedly decreased at 1 and $3 \mathrm{~h}$ post-MCAO, but was increased at $9 \mathrm{~h}$, without reaching 

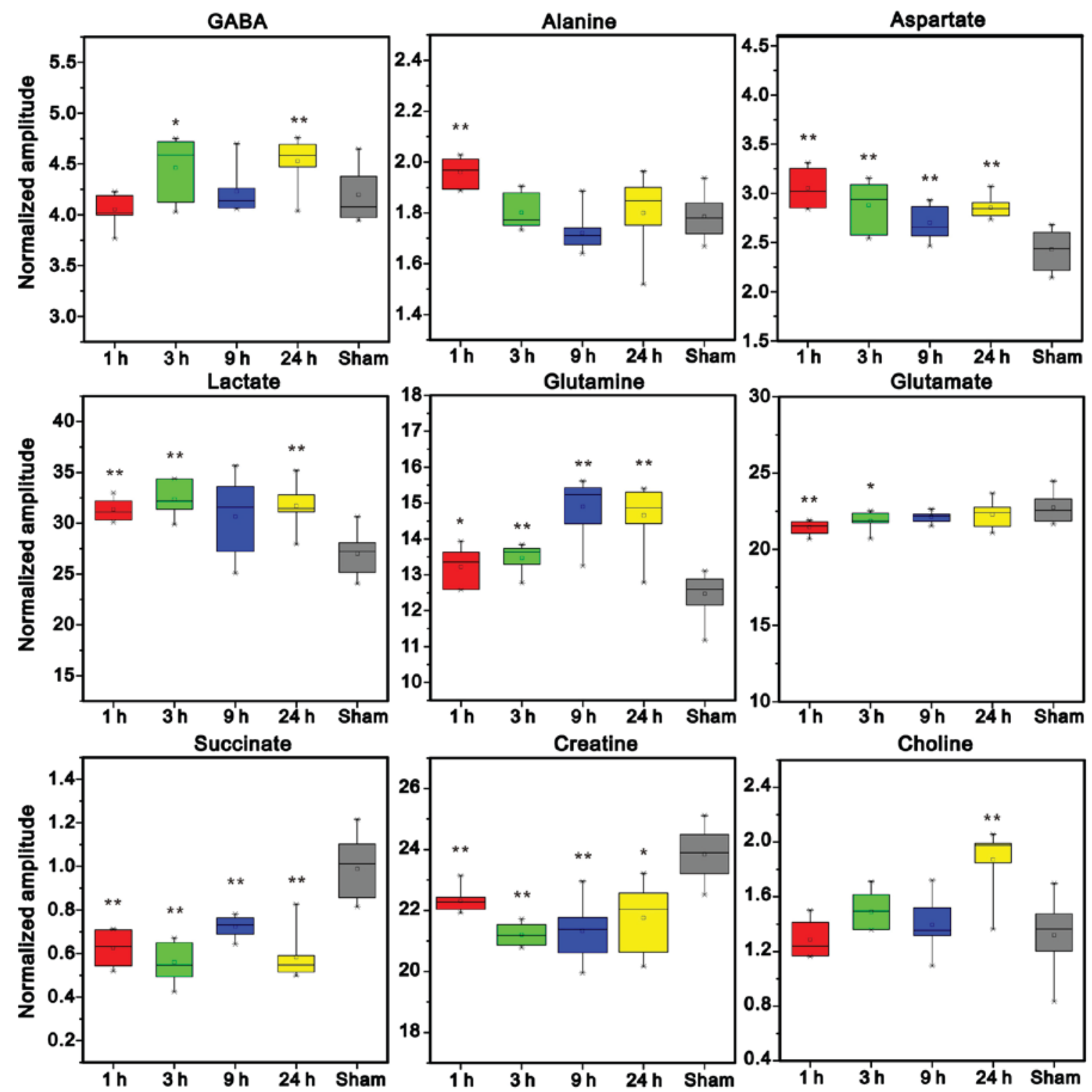

Figure 9. Relative normalized concentrations of the significantly altered metabolites in the right cerebellum. Red, green, blue, yellow and grey bar charts represent relative normalized concentrations in the middle cerebral artery occlusion 1,3,9 and $24 \mathrm{~h}$, compared with sham operation groups, respectively. ${ }^{*} \mathrm{P}<0.05 ;{ }^{* * *} \mathrm{P}<0.01$ vs. the sham group. GABA, $\gamma$-aminobutyric acid.

statistical significance. In addition, according to the PLS-DA score plots, as the duration of ischemia increased, the metabolic pattern in the left cerebral hemisphere moved gradually away from the sham group along the $t(1)$ direction; however, this was not the case for the metabolic pattern in the right cerebellum at $9 \mathrm{~h}$. It may be hypothesized that Glu was not transferred from the non-ischemic right cerebellum to the left ischemic brain at $9 \mathrm{~h}$ post-MCAO. As a result, the excitotoxic burden in the ischemic region is decreased. This finding also suggested that CCD is reversible $9 \mathrm{~h}$ after ischemic injury. The metabolic alterations of Glu may be associated with CCD. It has previously been reported that following permanent ischemia, metabolic alterations can be longitudinally followed using in vivo localized ${ }^{1} \mathrm{H}$ MRS $(39,40)$. Berthet et al (40) detected marked metabolic alterations in the ischemic core following permanent focal ischemia, including increases in GABA, Gly and Cre, and decreases in Gln, Glu and NAA. However, the results of the present study, including the increases in Gln levels in the ischemic cerebral hemisphere, were not consistent with the previous in vivo results. Further studies are required to assess these inconsistencies.

NAA is regarded as a sensitive marker of neuronal function (41). The levels of NAA were significantly decreased in the ischemic cerebral hemisphere of rats. This indicated that the ischemic brain damage may lead to obvious neuronal dysfunction. Cre is a biological marker for the energy metabolism of neurons $(42,43)$. In the present study, the levels of Cre were markedly decreased in the left cerebral hemisphere and the right cerebellum, which revealed that energy metabolism of the brain was disordered in the ischemic rats. Cho is involved in lipid metabolism and membrane function (44). An increase in the levels of Cho in the brain suggests a significant alteration in membrane metabolism caused by ischemia. In addition, the reduction in the levels of $\mathrm{m}$-Ins detected in the present study was consistent with previous findings (45) and may be a suggestive of alterations in the local osmotic pressure of cells induced by the ischemia. 

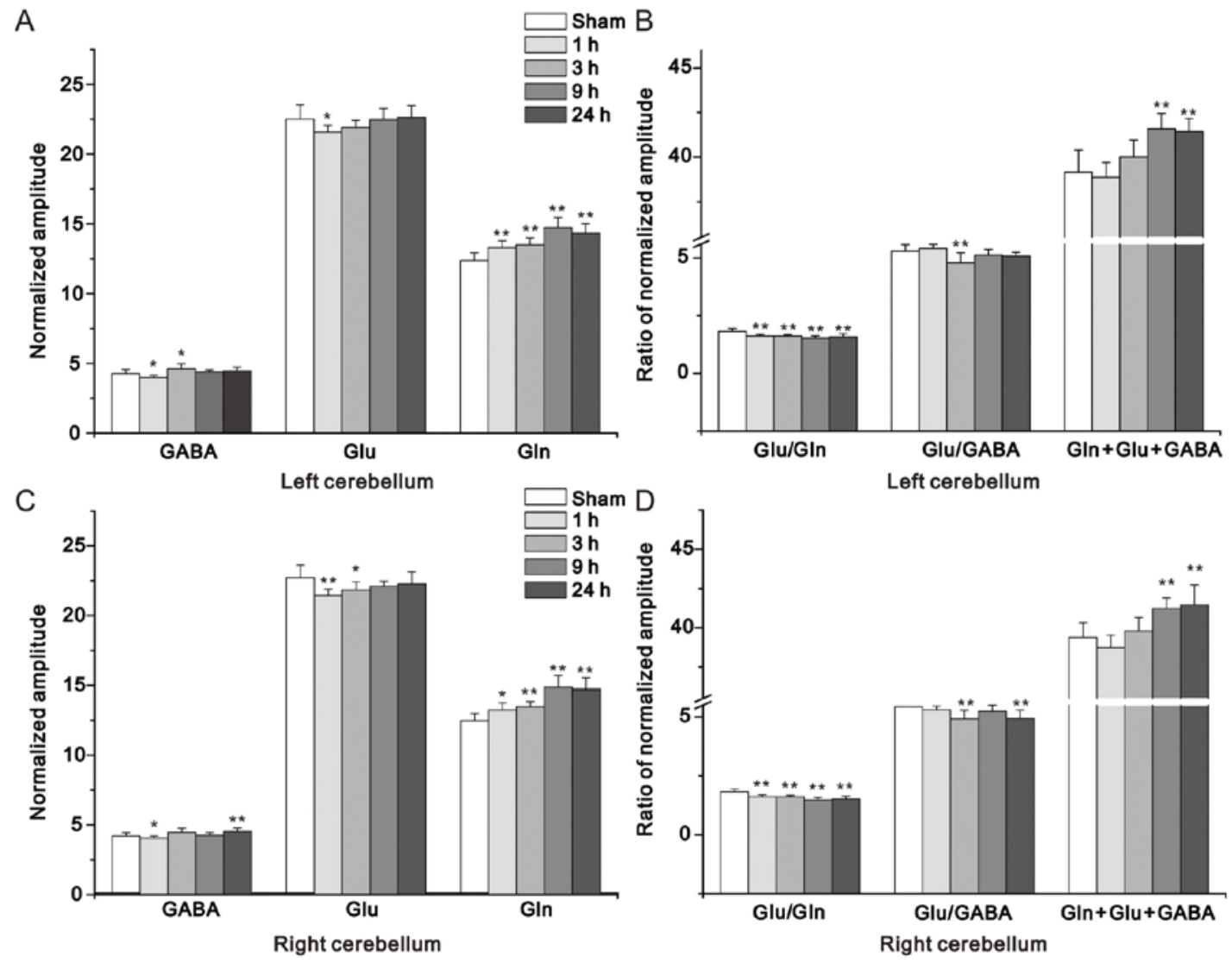

Figure 10. Summed concentration of Glu, Gln, GABA, Glu/Gln ratio, Glu/GABA ratio and Gln + Glu + GABA in (A and B) the non-ischemic left cerebellum and $(\mathrm{C}$ and $\mathrm{D})$ right cerebellum in the 1,3,9 and $24 \mathrm{~h}$ middle cerebral artery occlusion, compared with sham groups. ${ }^{*} \mathrm{P}<0.05 ;{ }^{* *} \mathrm{P}<0.01$ vs. the sham group. GABA, $\gamma$-aminobutyric acid; m-Ins, myo-inositol; Gln, glutamine; Glu, glutamate.

The effects of ischemia on metabolism in the ischemic cerebral hemisphere and in other brain regions have been reported in a previous ex vivo analysis (1) and in an in vivo study (46). However, some of the findings from the ischemic cerebral hemisphere in the present study were inconsistent with the findings of other studies regarding permanent MCAO $(1,39,40,47)$, which may be due to numerous factors, as listed below.

Firstly, there are differences between the methods used to generate successful models of ischemia, including the control of cerebral blood circulation for filament insertion-induced focal ischemia (5). This may result in differences in the metabolic response to permanent ischemia in the cerebral cortex, consequently resulting in increased variability in metabolic results $(21,39,40)$.

Secondly, fixation of cerebral tissue following decapitation has potential postmortem effects on Lac, Cre and GABA, which are key components used for PLS-DA in the present study. It is well known that fixation and extraction procedures intrinsically affect metabolic results. For example, decapitation is known to induce postmortem effects on metabolism $(2,48,49)$. Typically, highly elevated Lac is expected following decapitation, due to the degradation of glucose and glycogen in the brain, and may also occur during extraction procedures. In addition, GABA is known to increase and the levels of phosphocreatine are known to immediately diminish after decapitation (48). The effects of fixation following decapitation have also been observed in vivo in the ischemic core following permanent ischemia using localized ${ }^{1} \mathrm{H}$ MRS in a horizontal $600 \mathrm{MHz}$ magnet (40). Therefore, the majority of the significant metabolic alterations induced by permanent ischemia in the present study may have been affected by the fixation method used; for example, highly elevated Lac levels were detected in MCAO rats compared with in sham-operated rats and therefore the difference in Lac between sham-operated and ischemic rats was significantly reduced. In addition, a three-fold elevation of GABA (40) in vivo following permanent ischemia was reduced; however, the difference was not so marked ex vivo in the present study. Alternatively, microwave fixation (50) and funnel-freeze fixation (51), with careful extraction procedures, have been reported to reserve all carbohydrates, including glucose and glycogen, as illustrated by the lower Lac levels detected in the control animals.

Finally, the selection of specimens may have effects on the results. For example, the effects of focal ischemia were limited to only part of one side of the cerebral hemisphere in Igarashi et al (26), Berthet et al (40) and Håberg et al (1). Therefore, the results from the selected specimen (part of the left cerebral hemisphere) would intrinsically provide information regarding the metabolic alterations in the ischemic cerebral hemisphere and in some non-ischemic regions. This may explain the discrepancies between the metabolic alterations detected in the present study compared with other studies $(1,39,40,47)$.

In conclusion, the present study used ${ }^{1} \mathrm{H}$ NMR-based metabonomics to evaluate cerebral metabolism in the ischemic cerebral hemisphere and the non-ischemic cerebellum post-MCAO in rats. The results indicated that focal ischemia affected non-ischemic cerebellum activities, neurotransmitter 
synthesis and metabolic balance, particularly in the contralateral cerebellum. In addition, metabolic activity in the cerebellum, particularly with regards to Glu, may serve an important role in brain function reconstruction, which may help improve understanding regarding cerebral infarction on a molecular level. Alterations in Glu metabolism may be associated with $\mathrm{CCD}$; however, this requires further experimentation.

\section{Acknowledgements}

The present study was supported by the Fund of Zhejiang Provincial Key Laboratory of Aging and Neurological Disorder Research (grant nos. 2012E10008 and LH001); the National Natural Science Foundation of China (grant nos. 81571626 and 21575105); the Natural Science Foundation of Zhejiang Province (grant no. LY15H220001); the Medical Science and Technology Project of Zhejiang Province (grant no. 2014KYA134); and the Science and Technology Planning Project of Wenzhou City (grant no. Y20140731).

\section{References}

1. Håberg $\mathrm{AK}, \mathrm{Qu} \mathrm{H}$ and Sonnewald U: Acute changes in intermediary metabolism in cerebellum and contralateral hemisphere following middle cerebral artery occlusion in rat. J Neurochem 109 (Suppl 1): S174-S181, 2009.

2. Baron JC, Bousser MG, Comar D and Castaigne P: 'Crossed cerebellar diaschisis' in human supratentorial brain infarction. Trans Am Neurol Assoc 105: 459-461, 1981.

3. Pantano P, Baron JC, Samson Y, Bousser MG, Derouesne C and Comar D: Crossed cerebellar diaschisis. Further studies. Brain 109: 677-694, 1986.

4. Meyer JS, Obara K and Muramatsu K: Diaschisis. Neurol Res 15: 362-366, 1993.

5. Gold L and Lauritzen M: Neuronal deactivation explains decreased cerebellar blood flow in response to focal cerebral ischemia or suppressed neocortical function. Proc Natl Acad Sci USA 99: 7699-7704, 2002.

6. Rubin G, Levy EI, Scarrow AM, Firlik AD, Karakus A, Wechsler L, Jungreis CA and Yonas H: Remote effects of acute ischemic stroke: A xenon CT cerebral blood flow study. Cerebrovasc Dis 10: 221-228, 2000.

7. Meneghetti G, Vorstrup S, Mickey B, Lindewald $\mathrm{H}$ and Lassen NA: Crossed cerebellar diaschisis in ischemic stroke: A study of regional cerebral blood flow by $133 \mathrm{Xe}$ inhalation and single photon emission computerized tomography. J Cereb Blood Flow Metab 4: 235-240, 1984.

8. Ito H, Kanno I, Shimosegawa E, Tamura H, Okane $\mathrm{K}$ and Hatazawa J: Hemodynamic changes during neural deactivation in human brain: A positron emission tomography study of crossed cerebellar diaschisis. Ann Nucl Med 16: 249-254, 2002.

9. Liu Y, Karonen JO, Nuutinen J, Vanninen E, Kuikka JT and Vanninen RL: Crossed cerebellar diaschisis in acute ischemic stroke: A study with serial SPECT and MRI. J Cereb Blood Flow Metab 27: 1724-1732, 2007.

10. Lin DD, Kleinman JT, Wityk RJ, Gottesman RF, Hillis AE, Lee AW and Barker PB: Crossed cerebellar diaschisis in acute stroke detected by dynamic susceptibility contrast MR perfusion imaging. AJNR Am J Neuroradiol 30: 710-715, 2009.

11. Madai VI, Altaner A, Stengl KL, Zaro-Weber O, Heiss WD, von Samson-Himmelstjerna FC and Sobesky J: Crossed cerebellar diaschisis after stroke: Can perfusion-weighted MRI show functional inactivation? J Cereb Blood Flow Metab 31: 1493-1500, 2011

12. Jeon YW, Kim SH, Lee JY, Whang K, Kim MS, Kim YJ and Lee MS; Brain Research Group: Dynamic CT perfusion imaging for the detection of crossed cerebellar diaschisis in acute ischemic stroke. Korean J Radiol 13: 12-19, 2012.

13. Chen S, Guan M, Lian HJ, Ma LJ, Shang JK, He S, Ma MM, Zhang ML, Li ZY, Wang MY, et al: Crossed cerebellar diaschisis detected by arterial spin-labeled perfusion magnetic resonance imaging in subacute ischemic stroke. J Stroke Cerebrovasc Dis 23: 2378-2383, 2014.
14. Kang KM, Sohn CH, Kim BS, Kim YI, Choi SH, Yun TJ, Kim JH, Park SW, Cheon GJ and Han MH: Correlation of asymmetry indices measured by arterial spin-labeling MR imaging and SPECT in patients with crossed cerebellar diaschisis. AJNR Am J Neuroradiol 36: 1662-1668, 2015.

15. Swanson RA, Sagar SM and Sharp FR: Regional brain glycogen stores and metabolism during complete global ischaemia. Neurol Res 11: 24-28, 1989.

16. Pouwels PJ and Frahm J: Regional metabolite concentrations in human brain as determined by quantitative localized proton MRS. Magn Reson Med 39: 53-60, 1998.

17. Duarte JM, Lei H, Mlynárik V and Gruetter R: The neurochemical profile quantified by in vivo $1 \mathrm{H}$ NMR spectroscopy. Neuroimage 61: 342-362, 2012

18. Emir UE, Auerbach EJ, Van De Moortele PF, Marjańska M, Uğurbil K, Terpstra M, Tkáč I and Oz G: Regional neurochemical profiles in the human brain measured by (1)H MRS at $7 \mathrm{~T}$ using local B(1) shimming. NMR Biomed 25: 152-160, 2012.

19. Szilágyi G, Vas A, Kerényi L, Nagy Z, Csiba L and Gulyás B: Correlation between crossed cerebellar diaschisis and clinical neurological scales. Acta Neurol Scand 125: 373-381, 2012.

20. Gao H, Dong B, Liu X, Xuan H, Huang Y and Lin D: Metabonomic profiling of renal cell carcinoma: High-resolution proton nuclear magnetic resonance spectroscopy of human serum with multivariate data analysis. Anal Chim Acta 624: 269-277, 2008.

21. Longa EZ, Weinstein PR, Carlson S and Cummins R: Reversible middle cerebral artery occlusion without craniectomy in rats. Stroke 20: 84-91, 1989.

22. Westerhuis JA, van Velzen EJ, Hoefsloot HC and Smilde AK: Multivariate paired data analysis: Multilevel PLSDA versus OPLSDA. Metabolomics 6: 119-128, 2010.

23. Cloarec O, Dumas ME, Trygg J, Craig A, Barton RH, Lindon JC, Nicholson JK and Holmes E: Evaluation of the orthogonal projection on latent structure model limitations caused by chemical shift variability and improved visualization of biomarker changes in 1H NMR spectroscopic metabonomic studies. Anal Chem 77: 517-526, 2005.

24. Gao H, Xiang Y, Sun N, Zhu H, Wang Y, Liu M, Ma Y and Lei H: Metabolic changes in rat prefrontal cortex and hippocampus induced by chronic morphine treatment studied ex vivo by high resolution 1H NMR spectroscopy. Neurochem Int 50: 386-394, 2007.

25. Katsura K, Rodriguez de Turco EB, Folbergrová J, Bazan NG and Siesjö BK: Coupling among energy failure, loss of ion homeostasis, and phospholipase $\mathrm{A} 2$ and $\mathrm{C}$ activation during ischemia. J Neurochem 61: 1677-1684, 1993.

26. Igarashi H, Kwee IL, Nakada T, Katayama Y and Terashi A: $1 \mathrm{H}$ magnetic resonance spectroscopic imaging of permanent focal cerebral ischemia in rat: Longitudinal metabolic changes in ischemic core and rim. Brain Res 907: 208-221, 2001.

27. Frykholm P, Hillered L, Långström B, Persson L, Valtysson J and Enblad P: Relationship between cerebral blood flow and oxygen metabolism, and extracellular glucose and lactate concentrations during middle cerebral artery occlusion and reperfusion: A microdialysis and positron emission tomography study in nonhuman primates. J Neurosurg 102: 1076-1084, 2005.

28. Brouns R, Sheorajpanday R, Wauters A, De Surgeloose D, Mariën P and De Deyn PP: Evaluation of lactate as a marker of metabolic stress and cause of secondary damage in acute ischemic stroke or TIA. Clin Chim Acta 397: 27-31, 2008.

29. Cvoro V, Wardlaw JM, Marshall I, Armitage PA, Rivers CS, Bastin ME, Carpenter TK, Wartolowska K, Farrall AJ and Dennis MS: Associations between diffusion and perfusion parameters, $\mathrm{N}$-acetyl aspartate, and lactate in acute ischemic stroke. Stroke 40: 767-772, 2009.

30. Bruhn H, Frahm J, Gyngell ML, Merboldt KD, Hänicke W and Sauter R: Cerebral metabolism in man after acute stroke: New observations using localized proton NMR spectroscopy. Magn Reson Med 9: 126-131, 1989.

31. Wardlaw JM, Marshall I, Wild J, Dennis MS, Cannon J and Lewis SC: Studies of acute ischemic stroke with proton magnetic resonance spectroscopy: Relation between time from onset, neurological deficit, metabolite abnormalities in the infarct, blood flow, and clinical outcome. Stroke 29: 1618-1624, 1998.

32. Federico F, Simone IL, Lucivero V, Giannini P, Laddomada G, Mezzapesa DM and Tortorella C: Prognostic value of proton magnetic resonance spectroscopy in ischemic stroke. Arch Neurol 55: 489-494, 1998. 
33. Pereira AC, Saunders DE, Doyle VL, Bland JM, Howe FA Griffiths JR and Brown MM: Measurement of initial N-acetyl aspartate concentration by magnetic resonance spectroscopy and initial infarct volume by MRI predicts outcome in patients with middle cerebral artery territory infarction. Stroke 30: 1577-1582, 1999.

34. Graham SH, Chen J, Sharp FR and Simon RP: Limiting ischemic injury by inhibition of excitatory amino acid release. J Cereb Blood Flow Metab 13: 88-97, 1993.

35. Melani A, Pantoni L, Corsi C, Bianchi L, Monopoli A, Bertorelli R, Pepeu G and Pedata F: Striatal outflow of adenosine, excitatory amino acids, gamma-aminobutyric acid, and taurine in awake freely moving rats after middle cerebral artery occlusion: Correlations with neurological deficit and histopathological damage. Stroke 30: 2448-2455, 1999.

36. Iltis I, Koski DM, Eberly LE, Nelson CD, Deelchand DK Valette J, Ugurbil K, Lim KO and Henry PG: Neurochemical changes in the rat prefrontal cortex following acute phencyclidine treatment: An in vivo localized (1)H MRS study. NMR Biomed 22: 737-744, 2009.

37. Håberg A, Qu H, Haraldseth O, Unsgård G and Sonnewald U: In vivo injection of [1-13C]glucose and [1,2-13C]acetate combined with ex vivo 13C nuclear magnetic resonance spectroscopy: A novel approach to the study of middle cerebral artery occlusion in the rat. J Cereb Blood Flow Metab 18: 1223-1232, 1998.

38. Barbaresi P, Fabri M, Conti F and Manzoni T: D- $[3 \mathrm{H}]$ aspartate retrograde labelling of callosal and association neurones of somatosensory areas I and II of cats. J Comp Neurol 263: 159-178, 1987.

39. Gyngell ML, Busch E, Schmitz B, Kohno K, Back T, Hoehn-Berlage $M$ and Hossmann KA: Evolution of acute focal cerebral ischaemia in rats observed by localized $1 \mathrm{H}$ MRS, diffusion-weighted MRI, and electrophysiological monitoring. NMR Biomed 8: 206-214, 1995.

40. Berthet C, Xin L, Buscemi L, Benakis C, Gruetter R, Hirt L and Lei $\mathrm{H}$ : Non-invasive diagnostic biomarkers for estimating the onset time of permanent cerebral ischemia. J Cereb Blood Flow Metab 34: 1848-1855, 2014.
41. Demougeot C,Marie C, Giroud M and Beley A:N-acetylaspartate: A literature review of animal research on brain ischaemia. J Neurochem 90: 776-783, 2004.

42. Miller BL: A review of chemical issues in 1H NMR spectroscopy: $\mathrm{N}$-acetyl-L-aspartate, creatine and choline. NMR Biomed 4: 47-52, 1991.

43. Li S, Huang M, Wang X, Wang X, Chen F, Lei H and Jiang F: Retinal metabolic changes in an experimental model of optic nerve transection by ex vivo $1 \mathrm{H}$ magnetic resonance spectroscopy. Neurochem Res 36: 2427-2433, 2011.

44. Cecil KM and Jones BV: Magnetic resonance spectroscopy of the pediatric brain. Top Magn Reson Imaging 12: 435-452, 2001

45. Yang M, Wang S, Hao F, Li Y, Tang H and Shi X: NMR analysis of the rat neurochemical changes induced by middle cerebral artery occlusion. Talanta 88: 136-144, 2012.

46. Alf MF, Lei H, Berthet C, Hirt L, Gruetter R and Mlynarik V: High-resolution spatial mapping of changes in the neurochemical profile after focal ischemia in mice. NMR Biomed 25: 247-254, 2012.

47. Nonaka M, Yoshimine T, Kohmura E, Wakayama A, Yamashita T and $\mathrm{T} \mathrm{H}$ : Changes in brain organic osmolytes in experimental cerebral ischemia. J Neurol Sci 157: 25-30, 1998.

48. Lowry OH, Passonneau JV, Hasselberger FX and Schulz DW: Effect of ischemia on known substrates and cofactors of the glycolytic pathway in brain. J Biol Chem 239: 18-30, 1964.

49. Petroff OA, Ogino T and Alger JR: High-resolution proton magnetic resonance spectroscopy of rabbit brain: Regional metabolite levels and postmortem changes. J Neurochem 51: 163-171, 1988.

50. Kong J, Shepel PN, Holden CP, Mackiewicz M, Pack AI and Geiger JD: Brain glycogen decreases with increased periods of wakefulness: Implications for homeostatic drive to sleep. J Neurosci 22: 5581-5587, 2002.

51. Cruz NF and Dienel GA: High glycogen levels in brains of rats with minimal environmental stimuli: Implications for metabolic contributions of working astrocytes. J Cereb Blood Flow Metab 22: 1476-1489, 2002. 